\title{
Human Identification On the basis of Gaits Using Time Efficient Feature Extraction and Temporal Median Background Subtraction
}

\author{
Sadaf Asif \\ University Of Engineering and Technology, Taxila Pakistan \\ sadaf_wah@yahoo.com \\ Ali Javed \\ University Of Engineering and Technology, Taxila Pakistan \\ ali.javed@uettaxila.edu.pk \\ Muhammad Irfan \\ University Of Engineering and Technology, Taxila Pakistan \\ irfan29wah@yahoo.com
}

\begin{abstract}
Gait analysis is basically referred to study of human locomotion. From the surveillance point of view behavioral biometrics and recognition at a distance are becoming more popular in researchers rather than interactive and Physiological biometrics. In this paper, a time efficient Human gait identification system is proposed. Initially Human silhouettes are extracted by using temporal median background subtraction on video frames, which successfully removes shadows and models even complex background, proposed gait algorithm extracts contours from foreground silhouettes images and then three bounding boxes are drawn around contoured human image 1) upper part for arms movement 2) middle part for thigh and knee angles 3) Lower part for legs movement, knee and ankle angles. Gait cycles are extracted to find gait period and to take final decision for gait features selection, which is used for training. Thigh, Knee, Ankle angles and bounding boxes' widths are used as gait signatures but middle portion of human contains less variations of width in gait cycle hence computing efficiency can be achieved by ignoring width factor of middle part. SVM based training and identification is performed on extracted gait features. The proposed system is assessed using publicly available gait datasets and some indoor experimental videos created for this research work. The results reveal that the proposed algorithm is able to achieve an outstanding recognition rate.
\end{abstract}

Index Terms - Human Identification, CCTV, gait Analysis, SVM, Bounding Box, Contours.

\section{INTRODUCTION}

Behavioral biometric is gaining interest among researchers because it doesn't involve interaction with system or camera unlike interactive and Physiological systems like iris, fingerprints, face recognition, hands scanning. Gait identification has more advantages over other behavioral biometrics (e.g. keystroke-scan, speech recordings) like (1) Suspect can be trapped at a distance before it really becomes threat. (2) It is difficult to conceal someone's gaits. (3) It can be done inconspicuously using hidden camera or closed-circuit television (CCTV) cameras. (4) It can be done even with low quality videos. (5) Real time video processing for Human gait extraction and identification can be feasibly performed on ordinary system.

Gait Applications can be used in medical diagnostics for pathological gait, gait disorder, study of movement of animals. Biometric identification and forensic can be used at airports, banks or any other high security places.

Human Gait features can be signified in model free (appearance based, holistic) or model based manner. Model free approaches use features like velocity, shape, color model, texture and position for prediction or approximation. These techniques use gait masks, PCA and Canonical Analysis, motion description variations of a subject, self-similarity plots and clustering analysis, matrix representation, wavelet transform, radon transform [1] and Gait Energy Image [2]. Model free approaches are simple and fast but gait dynamics are indirectly linked with silhouette holistic information. For Model based approaches pre knowledge of object under consideration is required in video frames. It basically gathers gait dynamics for human transition characteristics like angular trajectory, shape style, geometric transformation, and body pose by directly modeling the basic kinematics of human motion. Model based techniques use three types of representations (1) blob (2) skeletal (3) Ribbon. Some Earlier work on it uses hip and thigh angular motion using Fourier series and 3D reconstruction of human poses [3].The tradeoff though is that these techniques 
are generally more multifaceted and require expensive computations and also need accurate extraction of human from background. Enormous research is being done on preprocessing (background subtraction, noise removal, changing lightning conditions, shadow removal) and some other factors like increase in processor power, decrease in price of memory and storage. These factors contribute to use model based tactic for reliable extraction of gait features.

In this research a new technique is introduced which uses both stagnant and kinematic features of human gait. Stagnant features from human structure are extracted like width of different body portions during walk, stride length and height. Kinematic features are represented by joint angles like thigh knee and ankles. This approach is not exactly model based because it does not model the human body; it only monitors the silhouette width and angles by analyzing the variations in succeeding strides. Beside legs movement during gait cycle, arms swing also has major contribution for identification of person. so keeping this factor in focus, for identification human body is divided in to three parts 1) arms 2) thigh 3) legs portion. Width variations caused by arms and legs movement are enormous than thigh portion of body, in order to save processing time, width factor of middle part is ignored but information of this part is still useful for thigh angle. Training and Classification are done with Support Vector Machine (SVM). The algorithm is implemented in Visual $\mathrm{C}$ and OpenCV while evaluated on some publicly available databases and videos captured by our own indoor video setups.

This Paper is structured in five sections. In Section II , literature review of human identification techniques is presented which includes some recent research strategies proposed by different authors. The proposed algorithm is described in Section III , following the explanation of extensive details of further steps. Experimental setup and acquired results obtained are discussed in section IV, which includes evaluation of algorithm on different publicly available datasets videos and results comparison with other proposed techniques. Last section includes our conclusion about research and future work.

\section{LITERATURE REVIEW}

E Adeli-Mosabbeb [3] proposed model based approach for Human gait detection. For path invariant tracking; 3D configuration of gait and angles is used. Classification is performed using SVM.

A-Kale [4] proposed a view based approach in which lower dimensional vector sequence was generated and for each human continuous density HMM was designed. Gait features chosen for identification are width of outer contour of silhouettes where switched of stances are represented by transition probabilities, while stances are associated with HMM states. By using these transitions in stances training is performed by encoding of width vector as a trampled 5-D observation sequence. After finding Euclidean distances between current subject and trained subjects, probability of HMM for current subject is computed by using Viterbi algorithm.

C. Nandini [5] first extracts silhouette using segmentation technique, then bounding box is drawn around silhouette and finally width, height and diagonal area of bounding box is taken as feature vectors. Hough transform is applied on silhouette and single Hough peak is found. Classification is performed using sum rule.

Murat EKINCI [6] represents walking of human as a multiple sequences of individual templates and entire backdrop pixels in the bounding box are also considered. Periodicity is produced by analyzing silhouette data. Eigen space transformation supported by Principal Component Analysis (PCA) is applied on 1-D Signals and classification is performed using Mahalanobis distances in lower dimensional Eigen space. Final decision is made using fusion strategy.

Atsushi Mori [7] suggested a period based matching of gait trajectory in Eigen space designed for lowframe rate videos using phase synchronization. First normalized autocorrelation is verdict for gait silhouette sequence considering temporal axis, hence it is maximized to detect gait period. These sequences are represented as trajectory dimensions in the Eigen space and time prolonging is used for synchronization of gait phase on the basis of gait.

Liang Wang [8] proposed a statistical shape analysis method for human detection. After background subtraction silhouettes are represented by consecutive sequences of complex vector configurations over time and represented in common coordinate frame. Mean shape obtained by Procrustes shape analysis is selected as gait signature. Based on full procrustes distance measure; supervised pattern classification is performed

\section{PROPOSED SYSTEM}

In this research paper, five steps algorithm proposed which outlined in Fig 1. Input to system is video frames containing human walking across passage, in lateral view.

\section{A. PREPROCESSING AND HUMAN DETECTION ACTIVITIES}

After Video Capturing first relevant step of human detection is background subtraction. Non stationary objects can be extracted from scene by taking difference between contemporary frame and the constructed background model with continuous update [9]. $\Delta \boldsymbol{t}$ Denotes the rate at which original $n$ video frames are subsampled. $\mathrm{BG}^{\mathrm{t}}$ is the background model w.r.t to time $t$ while $S P$ denotes still points in frame. Median is updated by adding Distance from each sample point to Current point in their L-inf sum.

$\mathrm{BG}^{\mathrm{t}+\Delta \mathrm{t}}(\mathrm{SP})=\min _{\mathrm{i}=1 \ldots \mathrm{k}} \sum_{\mathrm{j}=1}^{\mathrm{k}} \operatorname{Distance}\left(\mathrm{x}_{\mathrm{i}}, \mathrm{x}_{\mathrm{j}}\right)$ 
where $x_{i}, x_{j} \in S$

$\mathrm{S}$ is the set of vectors at time $\mathrm{t}$ for points in frames of video in RGB color space and background estimation covered by object itself. While Distance is computed by finding L-inf distance between current value and median value in RGB color space.

$\operatorname{Dis}\left(\mathrm{x}_{\mathrm{i}}, \mathrm{x}_{\mathrm{j}}\right)=\max \left(\left|\mathrm{x}_{\mathrm{i}(\mathrm{R}, \mathrm{G}, \mathrm{B})}-\mathrm{x}_{\mathrm{j}(\mathrm{R}, \mathrm{G}, \mathrm{B})}\right|\right)$

After Subtraction outer Contours are obtained from video frames, these are in fact exterior boundary of white regions in binary image. After that Contours are filled for human silhouette representation.

Image Enhancement is performed by smoothing, Dilation and Erosion techniques. Smoothing is blurring which is used for further noise reduction; Gaussian filter with 3-by-3 kernel is used for this purpose.

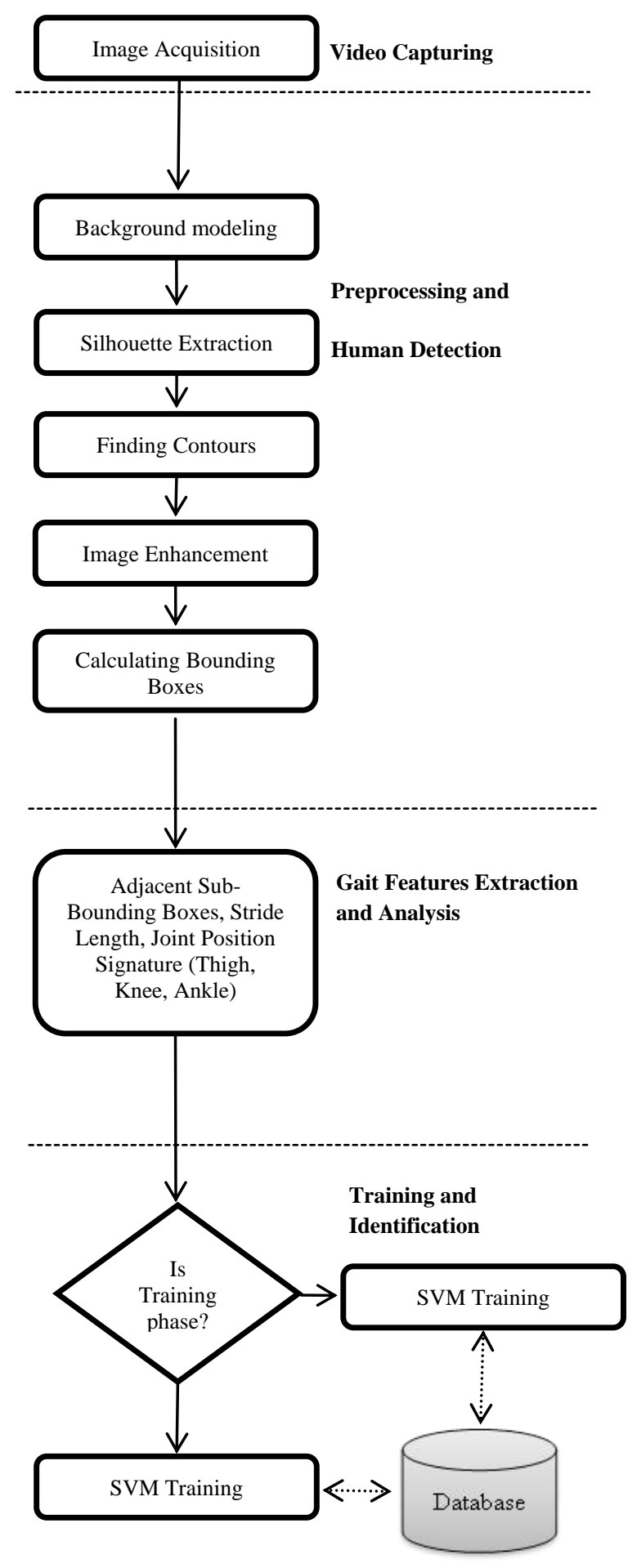

Figure 1. Flow Chart of Proposed algorithm 


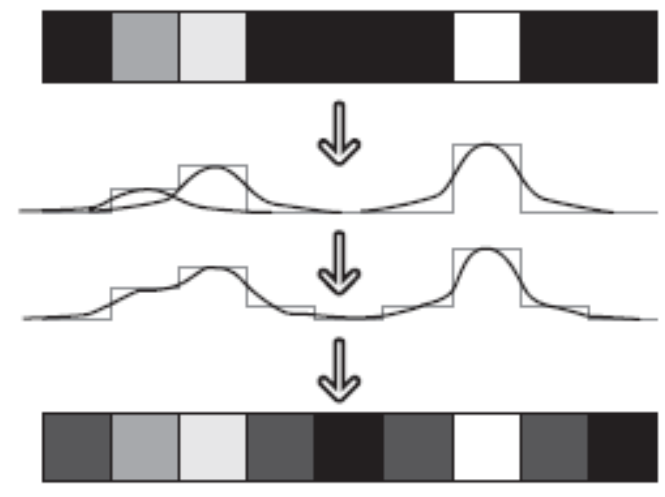

Figure 2. Gaussian Blur

Basic functionality of Dilation and Erosion is noise removal, Individual elements isolation, and disparate elements joining.

Dilation works by taking maximum from image A under kernel B as shown in Fig 3.

$$
\text { Dilate }(\mathrm{x}, \mathrm{y})=\max _{\left(\mathrm{x}^{\prime}, \mathrm{y}^{\prime}\right) \in \text { kernel }} \operatorname{src}\left(\mathrm{x}+\mathrm{x}^{\prime}, \mathrm{y}+\mathrm{y}^{\prime}\right)
$$

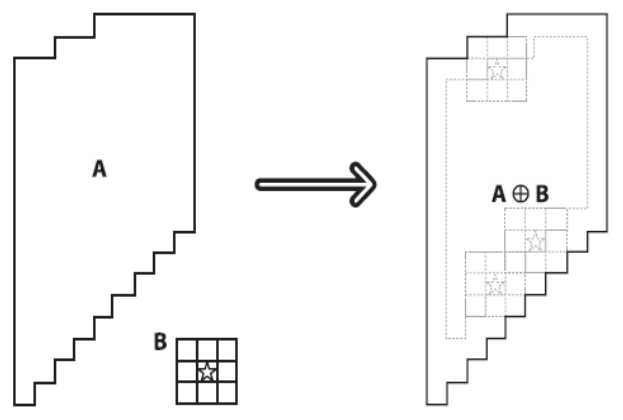

Figure 3. Dilation

Erosion works by taking minimum from image A under kernel B as shown in Fig 4.

$\operatorname{Erode}(x, y)=\min _{\left(x^{\prime}, y^{\prime}\right) \in \text { kernel }} \operatorname{src}\left(x+x^{\prime}, y+y^{\prime}\right)(4$

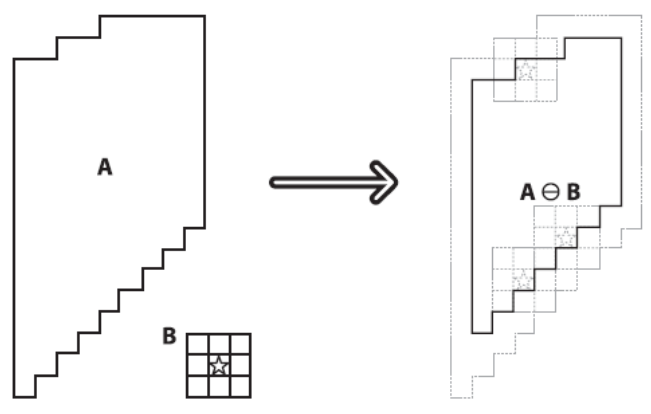

Figure 4. Erosion

Bounding Boxes drawn around silhouettes. Bounding box $B$ is minimum rectangular area which bound the filled contour [5].WB is width, and $W H$ is the height of $B$.

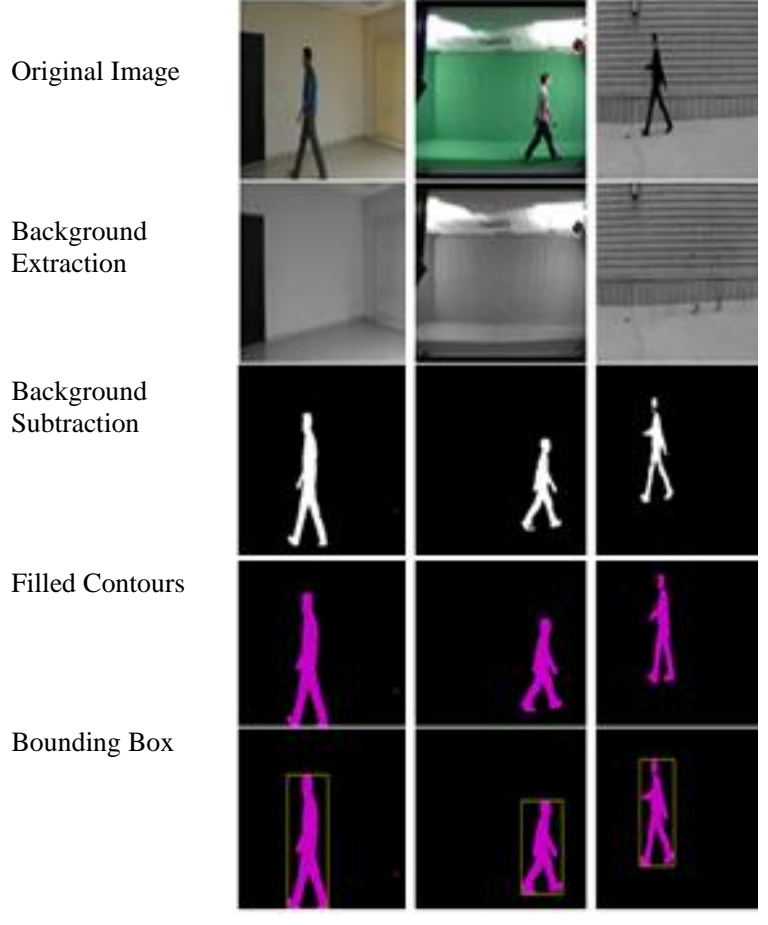

Figure 5. Human Detection Activities

\section{B. GAIT FEATURES EXTRACTION}

\section{1) Adjacent sub bounding boxes}

Silhouette width variation during gait cycle is important information for gait analysis because it represents both stagnant and kinematic features. Previous research on gait analysis suggests that arms swing distances are also important along legs swing distances [10] in gait cycle. So in our proposed technique human body divided in three parts (1) Upper (2) Middle (3) Lower. Results have shown that human middle part width variation is far less than upper and lower part, so upper and lower part's width taken as identifying gait features.

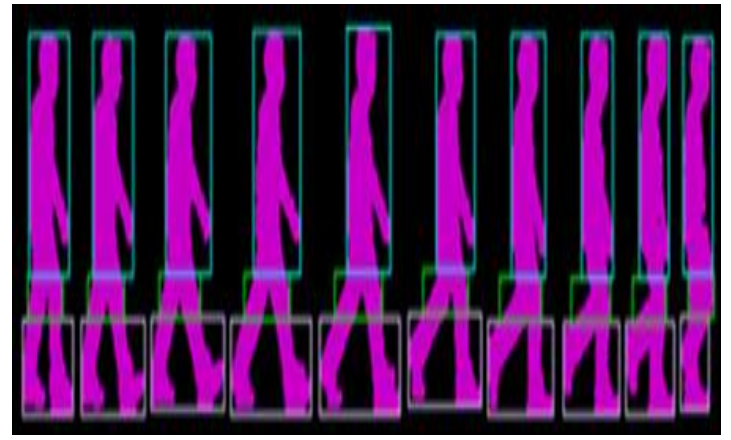

Figure 6. Adjacent sub-bounding boxes varying

Let's $W S_{1}, W S_{2}, W S_{3}$ be the width of sub bounding boxes respectively such that

$$
\mathrm{WB}=\max \left(\mathrm{WS}_{1}, \mathrm{WS}_{2}, \mathrm{WS}_{3}\right)
$$




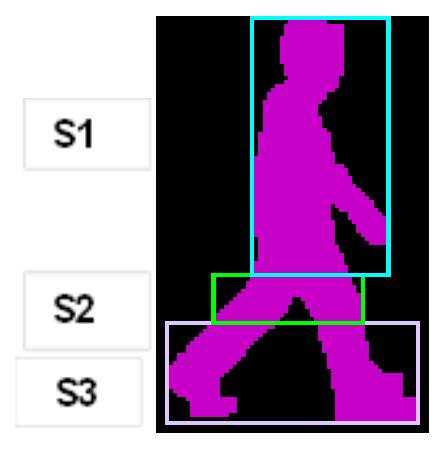

Figure 7. Adjacent sub bounding boxes width in one gait cycle

And heights of the sub bounding boxes are taken as

$\mathrm{WH}_{1}=0.25 * \mathrm{WH}$

$\mathrm{WH}_{2}=0.12 * \mathrm{WH}$

$\mathrm{WH}_{3}=\mathrm{WH}-\left(\mathrm{WH}_{1}+\mathrm{WH}_{2}\right)$

\section{2) Detection of Strides}

Single gait cycle is known as Stride. Stride is a period when person's foot touches the ground and then the same foot touches the ground again. Fig 8 shows human walk is periodical in nature, frame numbers are taken at $\mathrm{x}$-axis and Bounding Box width of silhouette is taken at $y$-axis.

In our case, by ignoring first cycle at least three consecutive cycles are extracted for better estimation of width and angles variation. Gait Period is extracted by taking average distance between two consecutive maxima peaks.

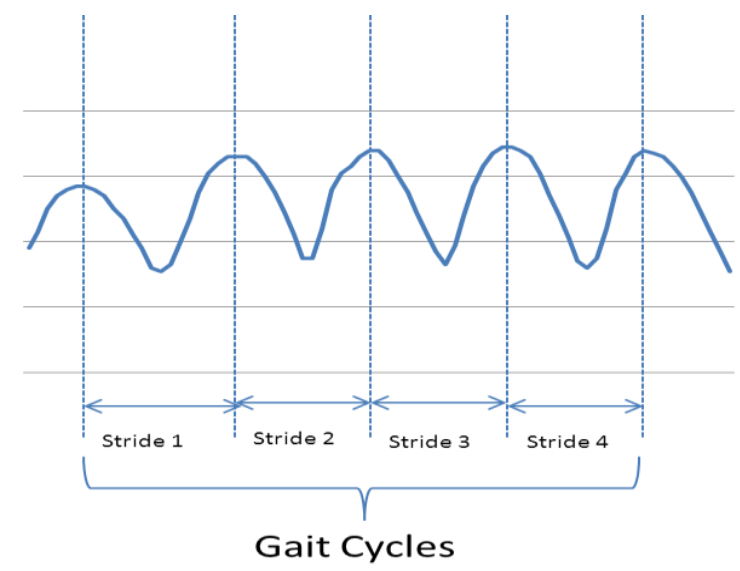

Figure 8. Gait Period Extraction by Finding Peaks

\section{3) Sub-bounding boxes Width extraction}

Average width of sub-bounding box $1 A V G W S_{1}$ is extracted by taking median of consecutive stride's width for upper part.

$\operatorname{AVG} \mathrm{WS}_{1}=\frac{1}{\mathrm{~N}} * \sum_{\mathrm{i}=0}^{\mathrm{N}} \operatorname{median}\left(\mathrm{WS}_{1}(\mathrm{t})\right)$
Average width of sub-bounding box $3 \mathrm{AVG} W S_{3}$ is extracted by taking median of consecutive stride's width for lower part.

$\mathrm{AVG} \mathrm{WS}_{3}=\frac{1}{\mathrm{~N}} * \sum_{\mathrm{i}=0}^{\mathrm{N}} \operatorname{median}\left(\mathrm{WS}_{3}(\mathrm{t})\right)$

Where $\boldsymbol{N}$ is the Number of strides considering here for Feature Extraction.

\section{4) Joint Position Signatures}

Varying Angles of legs over a period of time during person walk are building blocks for gait signatures as human walk is periodical in nature so these angles repeats themselves with slight difference each time.

Each person has unique walking style and hence their gait leads to extract unique combination of angles set during their walk cycle. So these angles can be stored for later identification of that human.

Here we are taking thigh, knee and ankle angles as gait signature for identification.

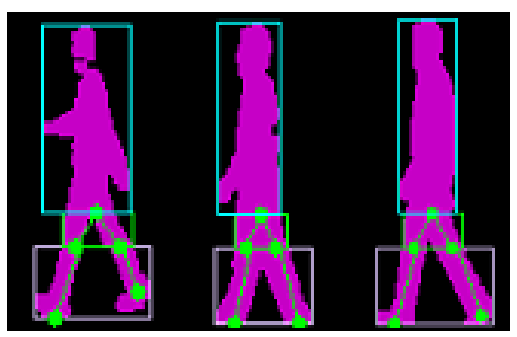

Figure 9. angles view for different humans at terminal swing phase

As Previous section shows human body is divided into three contiguous horizontal bounding boxes. Positions of joints are located to extract joint angles. Pelvis points $\left(X_{p}, Y_{p}\right)$ are found at $S_{2}$ by taking mid position at upper boundary. Front knee $\left(X_{k 1}, Y_{k 1}\right)$ and rear knee $\left(X_{k 2}, Y_{k 2}\right)$ points are located at $S_{3}$ where $X_{k 1}$ represents the horizontal position of left border of $S_{2}$ and $X_{k 2}$ denotes the right border of $S_{2}$. Front Ankle $\left(X_{A 1}, Y_{A 1}\right)$ is located at lower boundary of $S_{3}$ at left border while rear ankle $\left(X_{A 2}, Y_{A 2}\right)$ is found at right vertical border of $S_{3}$.

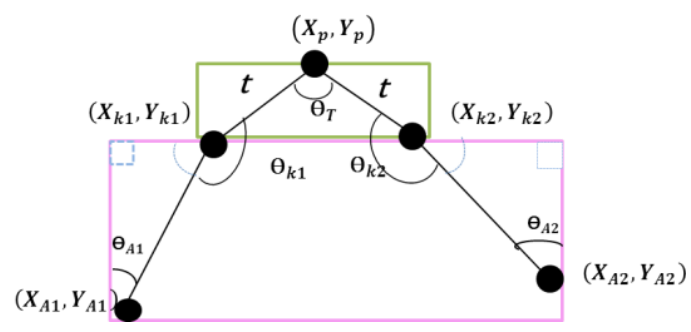

Figure 10. Angles Extraction

Thigh angle $\Theta_{T}$ is computed from Isosceles Triangle as

$\Theta_{\mathrm{T}}=\cos ^{-1}\left(1-0.5\left(\frac{w s_{2}}{t}\right)^{2}\right.$ 
While $\boldsymbol{t}$ and ankle angles $\Theta_{A 1}$ and $\Theta_{A 2}$ computed using right triangle formulas. Knee angles $\Theta_{k 1}$ and $\Theta_{k 2}$ as,

$$
\begin{aligned}
& \theta_{\mathrm{K} 1}=180+\theta_{\mathrm{A} 1}-\frac{\theta_{\mathrm{T}}}{2} \\
& \theta_{\mathrm{K} 2}=180+\theta_{\mathrm{A} 2}-\frac{\theta_{\mathbf{T}}}{2}
\end{aligned}
$$

\section{CLASSIFICATION}

Support Vector Machine (SVM) is used for classification. SVM is kernel based method which enable mapping of data from input space to higher dimension space making the possibility of separating hyper planes for multi classed data points. First Width vectors and joint position signatures are stored in database with person name as his ID and then SVM is trained by passing the feature vectors from database and their ID's as class labels for these vectors using linear kernel.

For Identification SVM prediction capability is used for current object features matching with trained support vectors which give ID of that human. Human name can be searched from database using that returned ID.

\section{EXPERIMENTAL SETUP AND RESULTS}

First we evaluate our proposed approach on our indoor video setup for lateral view walk. Than we test it on publicly available data sets like SOTON, Little \& Boyd, MIT, CMU Mob.

\section{A) GAIT DATABASE EVALUATION}

Human Identification on the basis of gait is challenging in biometric sciences but it is gaining interest among computer vision researchers because of its enormous advantages. Unavailability of an ascribed public database of reasonable size is a major limitation in the research of gait identification. Many papers present good recognition outcomes usually on small databases so direct comparison among papers is not possible but all results can be still overviewed.

We have distinguished between probe and gallery videos. Probe videos are used for training of SVM and gallery videos are used for prediction. We used the closed Universe method for evaluation which means we assume that each human in gallery is also present in probe videos.

As we have considered only lateral view so $0^{0}$ viewing angle videos from above mentioned datasets are selected and Rank1 results are shown.

1. Indoor setup specially created for this research having 10 subjects with 2 samples walks for each person. We have used one sample for training and other for testing.

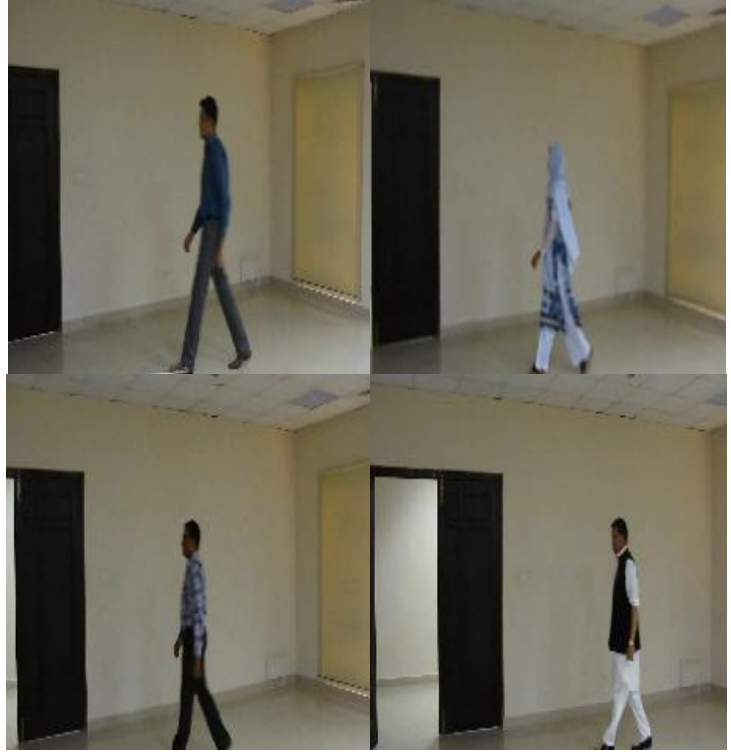

Figure 11. Some Sample video frames from Our Indoor database created for this research work.

TABLE 1. RESULT ON INDOOR DATABASE CREATED FOR THIS RESEARCH WORK

\begin{tabular}{|c|l|}
\hline Method & Results \\
\hline The Proposed Method & $100 \%$ \\
\hline
\end{tabular}

2. SOTON database is provided by University of Southampton, made by creating an indoor setup in uniform green background which enables to extract vibrant silhouettes.

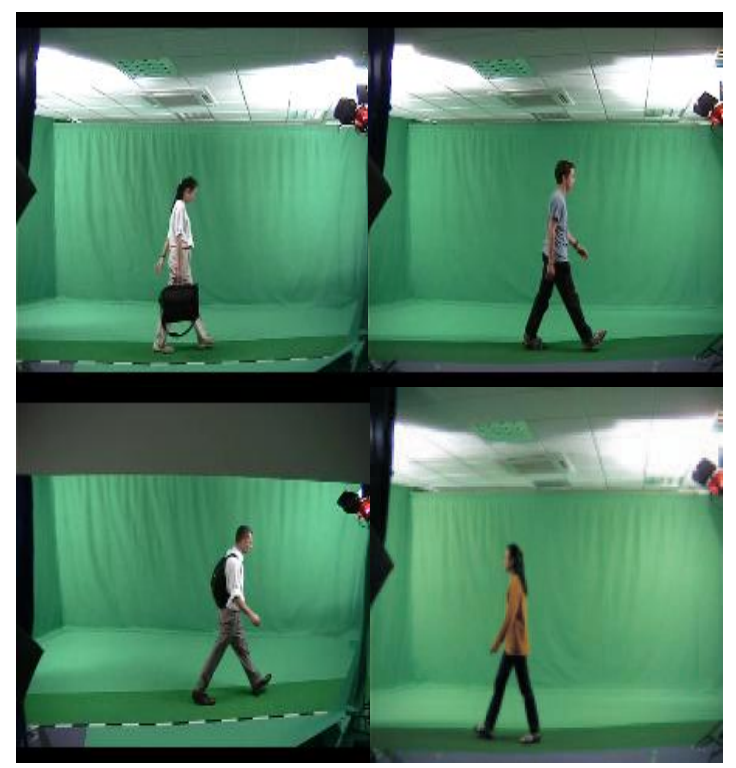

Figure 12. Some Sample video frames from SOTON database. 
TABLE 2. ALGORITHMS COMPARISON ON SOTON DATABASE

\begin{tabular}{|l|l|}
\hline \multicolumn{1}{|c|}{ Method } & Results \\
\hline Shutter 2000 [11] & $87.50 \%$ \\
\hline Hayfron-Acquah 2001[12] & $100 \%$ \\
\hline Foster 2001 [13] & $83 \%$ \\
\hline Laing 2003 [8] & $95.83 \%$ \\
\hline Beefing Goo 2009[14] & $86 \%$ \\
\hline Chen Wang 2012 [15] & $94 \%$ \\
\hline The Proposed Method & $100 \%$ \\
\hline
\end{tabular}

3. MIT database consists of 35 subjects with 4 motion sequences. Two of them from right to left and two from left to right. These Silhouettes video frames are of $640 \times 480$ size.

\section{TABLE 3. ALGORITHMS COMPARISON ON MIT} DATABASE

\begin{tabular}{|l|l|}
\hline Method & Results \\
\hline Nikolaos V. Boulgouris 2007 [1] & $87 \%$ \\
\hline Guoying Zhao 2009 [16] & $100 \%$ \\
\hline The Proposed Method & $98.50 \%$ \\
\hline
\end{tabular}

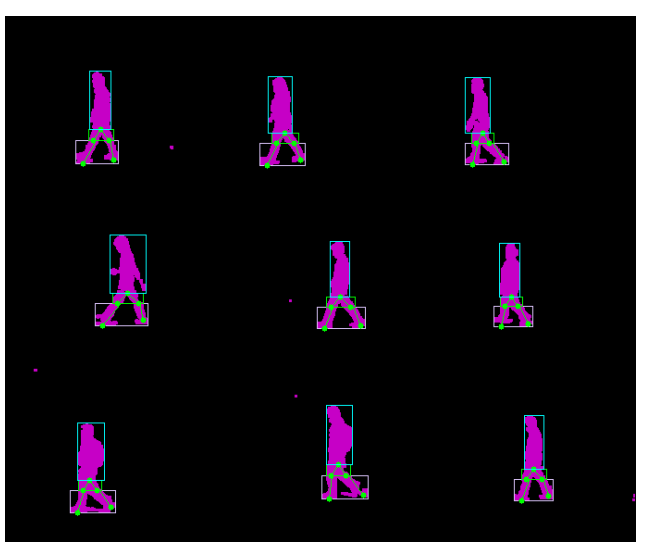

Figure 13. Some Sample Silhouette frames from MIT database

4. Little and Boyd consist of 5 different subjects having around 22 gait cycles. We have used its half cycles for training and half for testing.

TABLE. 4 ALGORITHMS COMPARISON ON LITTLE \& BOYD DATABASE

\begin{tabular}{|l|l|}
\hline Method & Results \\
\hline HAN SU [10] & $100 \%$ \\
\hline The Proposed Method & $100 \%$ \\
\hline
\end{tabular}

5. CMU MoBo dataset compromise of 25 subjects with 6 motion sequences walking on treadmill.
TABLE 5. ALGORITHMS COMPARISON ON CMU MOBO DATABASE FOR LATERAL VIEW

\begin{tabular}{|l|l|}
\hline Method & Results \\
\hline Nikolaos V. Boulgouris 2007 [1] & $87 \%$ \\
\hline Murat EKINCI 2006 [6] & $88 \%$ \\
\hline Sabesan Sivapalan 2011 [2] (intra class) & $100 \%$ \\
\hline Guoying Zhao 2009 [16] & $100 \%$ \\
\hline The Proposed Method & $100 \%$ \\
\hline
\end{tabular}

\section{CONCLUSION}

In this paper we have combined stagnant and kinematic features of human gait for identification. Temporal median background subtraction technique is chosen as preprocessor as it removes shadows and quickly models foreground from changing background objects in scene. Drawing of bounding boxes around parts of body gives width vector in which upper part of body shows arms swings and lower part indicates legs swing information. As middle part shows very minor variation in width so this part is ignored from width vector perspective. Joint angles are extracted from middle and lower parts and geometry basic formulas are used for angles calculation. For classification Support Vector Machine is used. The results comparisons with other published papers show that the proposed algorithm gives efficient recognition results, which shows its practicability. But still our database is small and we have not tested on random samples and proposed technique deals only with lateral view so the factors like mode swings, clothes variation and walking views will be covered as future task.

\section{REFERENCES}

[1] Nikolaos V. Boulgouris, Gait Recognition Using Radon Transform and Linear Discriminant Analysis. IEEE Transactions On Image Processing, Vol. 16, No. 3, March 2007.

[2] Sabesan Sivapalan, Daniel-Chen, Simon-Denman, SridhaSridharan and Clinton Fookes, Gait energy volumes and frontal gait recognition using depth images. International Joint Conference on Biometrics, IEEE, USA 2011.

[3] E Adeli-Mosabbeb, M Fathya and F Zargari, Model-based human gait tracking, 3D reconstruction and recognition in uncalibrated monocular video. Imaging Science Journal vol 0, 2011.

[4] A-Kale, A.N-Rajagopalan, N-Cuntoor and VKruger, Gait-based Recognition of Humans Using Continuous HMMs. IEEE Proceedings of the Fifth International Conference on Automatic Face and Gesture Recognition 2002.

[5] C.Nandini, PratulMukhopadhyay, An Efficient Human Identification Using Gait Analysis. International Journal of Research and Reviews in 
Computing Engineering (IJRRCE) Vol. 1, No. 2, June 2011.

[6] Murat Ekinci, Human Identification Using Gait. Turk J ElecEngin, VOL.14, NO.2 2006.

[7] Atsushi Mori, Yasushi Makihara, Yasushi Yagi, Gait Recognition using Period-based Phase Synchronization for Low Frame-rate Videos. International Conference on Pattern Recognition 2010 .

[8] L-Wang, T-Tan, W-Hu, Automatic Gait Recognition Based on Statistical Shape Analysis. IEEE Transactions On Image Processing, Vol. 12, No. 9, September 2003.

[9] Rita Cucchiara, Detecting Moving Objects, Ghosts, and Shadows in Video Streams. IEEE transactions on pattern analysis and machine intelligence, vol. 25, no. 10, October 2003.

[10] Han-Su, Feng-Gang Huang, Human Gait Recognition Based On Motion Analysis. Proceedings of the Fourth International Conference on Machine Learning and Cybernetics, Guangzhou, 18-21 August 2005.

[11] J-hutler,M. Nixon, C. Harris, Statistical gait recognition via temporal moments. In Proc. 4th IEEE Southwest Symp. Image Analysis and Interpretation, 2000, pp. 291-295.

[12] J. B-Hayfron-Acquah, M. S-ixon, and J. N. Carter, Automatic gait recognition by symmetry analysis. In Proc. 3rd Int. Conf. Audio- and Video-Based Biometric Person Authentication, 2001.

[13] J. Foster, M. Nixon, A. Prugel-Bennett, New area based metrics for gait recognition. In Proc. 3rd Int. Conf. Audio- and Video-Based Biometric Person Authentication, 2001.

[14] Baofeng Guo,Mark S. Nixon, Gait Feature Subset Selection by Mutual Information. IEEE Transactions on Systems, Man, and Cybernetics-Part A: Systems And Humans, Vol. 39, No. 1, January 2009.

[15] Chen Wang, Junping Zhang, Liang Wang, Human Identification Using Temporal Information Preserving Gait Template. In IEEE Transactions on Pattern Analysis and Machine Intelligence, Vol. 34, No. 11, November 2012.

[16] Guoying Zhao,Li Cui,Hua Li,Matti Pietikainen, Gait Recognition Using Fractal Scale and Wavelet Moments.Iin IEEE ICPR 2006.

[17] Tan, Tienjui N.; Hu, Weinming; Ning, Huazhong, Automatic Gait Recognition based on Statistical Shape Analysis, IEEE Transactions on Image Processing, Vol 12, Issue 9.

\section{AUTHOR'S BIOGRAPHY}

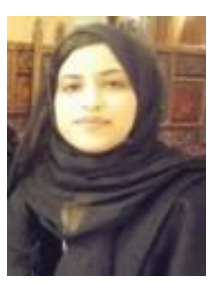

Engr. Sadaf Asif received her BSc degree from University of Engineering and Technology, Taxila, Pakistan in Software Engineering. She is currently working as research associate in University Of Engineering and Technology Taxila. Her current research interests include Computer Vision, Image Processing, Satellite Communication, and Software Development.

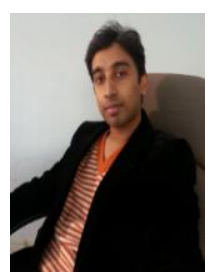

Engr. Ali Javed is serving as an Assistant Professor in the Department of Software Engineering at UET Taxila Pakistan. He is also a PhD Scholar in Computer Engineering Department at UET Taxila, Pakistan. He has received his MS degree in Computer Engineering from UET Taxila, Pakistan in February, 2010. He received Chancellor's Gold Medal in MS Computer Engineering degree and became the first MS student in the history of UET Taxila to be awarded Chancellor's Gold Medal. He has received B.Sc. degree in Software Engineering from UET Taxila, Pakistan, in September, 2007. He got 3rd position in Software Batch-2003F in BS Software Engineering degree. His areas of interest are Digital Image Processing, Computer vision, Video Summarization, Mobile Application Development, Software Requirements Engineering, Software Quality Assurance and Software testing.

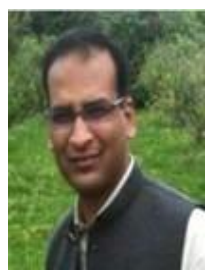

Dr Muhammad Irfan did his bachelor in Electrical Engineering in 2001 from University Of Engineering And Technology Taxila. He did his M.Sc Embedded system and control Enginnering in 2006 and P.hd Engineering in 2010 from Leicester university UK. His areas of interests are embedded system, control design, image processing and robotics. 